\title{
The Effect of Dezocine Combined with Oxycodone in the Treatment of Advanced Lung Cancer with Bone Metastasis and Severe Cancer Pain on the Numerical Rating Scale Score of Patients
}

\author{
CHUNMEI YAN AND YAN LIU* \\ Department of Critical Care Medicine, West China Hospital, Sichuan University, Chengdu, WenJiang 610041, China
}

Yan et al.: Effect of Dezocine Combined with Oxycodone

\begin{abstract}
The effect of dezocine combined with oxycodone on the pain score (numerical rating scale score) of patients with severe cancer pain caused by bone metastasis of advanced lung cancer was analyzed. The patients were divided into two groups with 50 cases in each group. The control group was given intramuscular injection of dezocine for analgesia and the observation group was given oral oxycodone hydrochloride controlled release tablets on the basis of intramuscular injection of dezocine for analgesia. The pain relief rate and analgesic efficiency of the observation group were better than those of the control group and the numerical rating scale score was lower than that of the control group. The number of burst pain in the observation group was less than that in the control group, the stable time of first pain control was shorter than that in the control group and the increase time of $\mathbf{2 4} \mathrm{h}$ sleep was longer than that in the control group, with statistical significance. After treatment, the overall health status score, functional score and symptomatic score of the two groups were significantly higher than those before treatment. Dezocine combined with oxycodone in the treatment of severe cancer pain in advanced lung cancer with bone metastasis can effectively relieve pain, regulate the expression of pain factors and improve the quality of life.
\end{abstract}

Key words: Dezocine, oxycodone, advanced lung cancer, adverse reactions

Lung cancer takes bronchial mucosa as the primary site and then transfers and spreads through bronchus, lymph and blood. Bone is the most common site of lung cancer metastasis. Bone metastasis is one of the common complications in patients with advanced lung cancer, which can cause osteolytic destruction, cancer pain and seriously affect the quality of life of patients ${ }^{[1]}$. The World Health Organization (WHO) proposed the three-step analgesic treatment principle. Mild pain: Non-steroidal anti-inflammatory drugs were given, moderate pain: weak opioid drugs were given and severe pain strong opioid drugs were given ${ }^{[2]}$. Dezocine is a potent opioid receptor agonist-antagonist, which is commonly used in clinical treatment of severe cancer pain. However, studies have found that some patients still have paroxysmal pain symptoms after using dezocine alone and the analgesic effect is not stable ${ }^{[3]}$.

Oxycodone is a pure opioid receptor agonist, which can act on opioid $\mu$ receptor and kappa ( $\kappa)$ receptor.
Oxycodone has analgesic effects on various pains and has more prominent effects on visceral pain and neuropathic pain. Studies have shown that the analgesic effect of oxycodone is more than twice that of morphine ${ }^{[4]}$. The difference between dezocine and oxycodone in the treatment of severe cancer pain in advanced lung cancer with bone metastasis is worthy of further discussion. In this study, the pain score Numerical Rating Scale (NRS) was used as a tool to analyze the effect of dezocine combined with oxycodone on the pain degree of patients with severe cancer pain caused by bone metastasis of advanced lung cancer. The reports are as follows.

\section{MATERIALS AND METHODS}

\section{General information:}

SA total of 100 patients from January 2020 to May 2021 were randomly divided into control group (50 cases) and observation group (50 cases). There were 
28 males and 22 females in the control group. The age was 35-75 y old, with an average of (62.04 \pm 9.82$)$ y old, Body Mass Index (BMI) $(22.52 \pm 2.06) \mathrm{kg} / \mathrm{m} 2$. The observation group had 26 males and 24 females, aged $33-75$ y old, with an average of $(60.98 \pm 10.05)$ y old, BMI $(22.45 \pm 2.24) \mathrm{kg} / \mathrm{m}^{2}$. Pathological type: adenocarcinoma 35 cases, squamous cell carcinoma 15 cases. Two groups of patients during the trial, no shedding, lost to follow-up cases, in the final completion of 100 cases. There was no significant difference in the above baseline data between the two groups, indicating comparability.

\section{Diagnostic standards:}

Western medicine: Reference (Diagnostic and therapeutic norms for primary lung cancer (2018 edition) [5] standard: with clear local growth performance of primary lung cancer. There has been a distant transfer. Pathological examination and immunohistochemistry confirmed non-small cell lung cancer and confirmed by imaging.

Inclusion of the standards: According to the standard of advanced lung cancer, the Union for International Cancer Control (UICC) belongs to stage IV and $\geq 1$ target lesion can be measured. Bone metastases occurred; Age $\geq 18$ y; patients with advanced lung cancer who failed in multi-line treatment were mainly supported; $\mathrm{NRS} \geq 8$; expected survival $>2$ mo.

Elimination standards: Heart, liver, renal insufficiency or disorder; other respiratory diseases; mental retardation or difficulty in judging the degree of pain; history of opioid abuse; allergic constitution.

\section{Treatment methods:}

The control group was given dezocine injection (Yangzijiang Pharmaceutical Group Co., Ltd., specification: $1 \mathrm{ml}: 5 \mathrm{mg}$, H20080329) intramuscular injection for analgesia, $10 \mathrm{mg} /$ time, once every $6 \mathrm{~h}$. The observation group was given oxycodone hydrochloride controlled release tablets (Mengti China Pharmaceutical Co., Ltd., specification $10 \mathrm{mg}$, J20140124 ) on the basis of intramuscular injection of dezocine for analgesia, oral $10 \mathrm{mg} /$ time, once every $12 \mathrm{~h}$. Both groups were treated for $14 \mathrm{~d}$.

\section{Observation index:}

The changes of NRS score and European Organisation for Research and Treatment of Cancer Quality Of Life Questionnaire (EORTC QLQ-C30) score were compared. The levels of beta-Endorphin $(\beta-E P)$ and 5 -Hydroxytryptamine or serotonin $(5-\mathrm{HT})$ were detected. The curative effect, safety, the number of 24 h pain outbreaks, the first time of pain control, stability time and $24 \mathrm{~h}$ sleep, increase time were observed.

\section{Mechanism exploration:}

Before treatment and $14 \mathrm{~d}$ after treatment, $2 \mathrm{ml}$ of fasting peripheral venous blood samples were collected and placed in Ethylenediamine Tetraacetic Acid (EDTA) anticoagulant tube. The samples were centrifuged within $2 \mathrm{~h}$ after blood collection and centrifuged at $4000 \mathrm{r} / \mathrm{min}$ for $10 \mathrm{~min}$. The upper serum was taken to detect $\beta$-EP and 5-HT by enzyme-linked immunosorbent assay kit (Shanghai Enzyme-linked Biotechnology Co., Ltd.). The detection instrument was RT-96A microplate reader (Shenzhen Mairui Medical Electronics Co., Ltd.).

\section{Efficacy criteria and scoring criteria:}

Reference NRS score decline rate assessment: Pretreatment score-Post treatment score)/cases $\times 100 \%$, decline $\geq 75 \%$ for significant remission; $50 \% \leq$ decrease $<75 \%$ is moderate remission; $25 \% \leq$ decrease $<50 \%$ is mild remission. Failure to meet the above criteria is non-relieving, significant, moderate and mild relief for pain.

NRS score; 0-3 points for mild pain, 4-7 points for moderate pain, 8-10 points for severe pain ${ }^{[6]}$.

EORTC QLQ-C30 score; it includes the overall health status field, functional field (5 items) and symptomatic field (9 items). The single score is based on the percentage system. The overall health status field and functional field are proportional to the quality of life and the symptomatic field is inversely proportional to the quality of life ${ }^{[7]}$.

\section{Statistical methods:}

Statistical Package For The Social Sciences (SPSS) 19.0 was used to process the data. The measurement index was described as $(\chi \pm s)$. Independent sample $t$ test was used for comparison between groups. Paired $\mathrm{t}$ test was used for comparison within groups. $\chi^{2}$ test was used for comparison of rates, $\mathrm{p}<0.05$ was statistically significant.

\section{RESULTS AND DISCUSSION}

Pain relief efficacy in the two groups was compared. The significant and moderate remission was 15 and 17, 12 mild, a pain relief rate of $88.00 \%$ and pain efficiency of $64.00 \%$, both superior to control and was statistically significant $(\mathrm{p}<0.05)($ Table 1$)$.

NRS scores of the two groups were compared. The 
pretreatment NRS score was similar $(\mathrm{p}>0.05)$, which decreased significantly compared with pretreatment $(\mathrm{p}<0.05)$ and lower after $1 \mathrm{~d}, 3 \mathrm{~d}, 7 \mathrm{~d}$ than in the control group ( $\mathrm{p}<0.05)$ (Table 2).

The number of $24 \mathrm{~h}$ bursts of pain, the time of $24 \mathrm{~h}$ sleep increase and the first stable time of pain control between the two groups were compared. Observation group $24 \mathrm{~h}$ bursts were less than the control, first pain control stabilization less than the control and $24 \mathrm{~h}$ sleep increased longer than the control $(\mathrm{p}<0.05)$ (Table 3$)$.

The two groups of $\beta$-EP, 5-HT were compared. Pretreatment $\beta$-EP, 5-HT was similar ( $p>0.05), \beta$-EP increased significantly before treatment $(p<0.05)$, 5 -HT lower before treatment $(\mathrm{p}<0.05)$ and higher after treatment in the observation group and 5-HT lower than the control group $(\mathrm{p}<0.05)$ (Table 4$)$.

EORTC QLQ-C30 scores of the two groups were compared. The EORTC QLQ-C30 scores before treatment were similar $(\mathrm{p}>0.05)$. After treatment, the scores of overall health status, function and nausea and vomiting in symptomatic areas of the two groups were significantly higher than those before treatment. The scores of pain, insomnia and loss of appetite in symptomatic areas were significantly lower than those before treatment. The scores of fatigue, shortness of breath, constipation, diarrhea and economic difficulties in symptomatic areas were similar to those before treatment ( $p>0.05)$. The scores of overall health status, function and nausea and vomiting in symptomatic areas of the observation group were higher than those of the control group. The scores of pain, insomnia and loss of appetite in symptomatic areas were lower than those of the control group. The scores of fatigue, shortness of breath, constipation, diarrhea and economic difficulties in symptomatic areas were similar to those of the control group ( $>>0.05)$ (Table 5).

TABLE 1: COMPARISON OF ANALGESIC EFFECTS BETWEEN THE TWO GROUPS [n (\%)]

\begin{tabular}{lccccccc}
\hline Group & $\begin{array}{c}\text { Number of } \\
\text { cases }\end{array}$ & $\begin{array}{c}\text { Significant } \\
\text { relief }\end{array}$ & $\begin{array}{c}\text { Moderate } \\
\text { remission }\end{array}$ & Mild relief & No remission & $\begin{array}{c}\text { Pain relief } \\
\text { rate }\end{array}$ & $\begin{array}{c}\text { Effective for } \\
\text { pain relief }\end{array}$ \\
\hline $\begin{array}{l}\text { Control group } \\
\text { Observation }\end{array}$ & 50 & 6 & 16 & 14 & 14 & $36(72.00)$ & $22(44.00)$ \\
group & 50 & 15 & 17 & 12 & 6 & $44(88.00)^{\#}$ & $32(64.00)^{\#}$ \\
\hline
\end{tabular}

Note: Compared with the controls, ${ }^{*} \mathrm{p}<0.05$

TABLE 2: COMPARISON OF NRS SCORES BETWEEN THE TWO GROUPS ( $\bar{x} \pm s, m i n)$

\begin{tabular}{lccccc}
\hline \multirow{2}{*}{ Group } & Number of cases & \multicolumn{3}{c}{ NRS score } \\
\cline { 3 - 6 } & & Before treatment & Treatment 1 d & Treatment 3 d & Treatment 7 d \\
\hline Control group & 50 & $8.89 \pm 0.46$ & $7.12 \pm 1.01^{*}$ & $6.03 \pm 0.67^{*}$ & $4.08 \pm 0.71^{*}$ \\
Observation group & 50 & $8.95 \pm 0.42$ & $6.33 \pm 0.90^{* \#}$ & $5.12 \pm 0.59^{* \#}$ & $2.26 \pm 0.54^{* \#}$ \\
\hline
\end{tabular}

Note: ${ }^{*} \mathrm{p}<0,0.05$; compared with the control group, ${ }^{\#} \mathrm{p}<0.05$

TABLE 3: COMPARISON OF THE NUMBER OF $24 \mathrm{~h}$ BURST PAIN, $24 \mathrm{~h}$ SLEEP INCREASE TIME AND FIRST

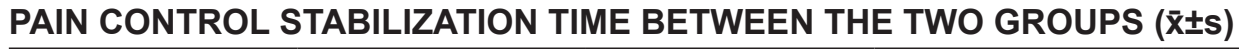

\begin{tabular}{lccccc}
\hline \multirow{2}{*}{ Group } & \multirow{2}{*}{ Number of cases } & \multicolumn{3}{c}{ NRS score } \\
\cline { 3 - 6 } & & Before treatment & Treatment 1 d & Treatment 3 d & Treatment 7 d \\
\hline Control group & 50 & $8.89 \pm 0.46$ & $7.12 \pm 1.01^{*}$ & $6.03 \pm 0.67^{*}$ & $4.08 \pm 0.71^{*}$ \\
Observation group & 50 & $8.95 \pm 0.42$ & $6.33 \pm 0.90^{* \#}$ & $5.12 \pm 0.59^{* \#}$ & $2.26 \pm 0.54^{* \#}$ \\
\hline
\end{tabular}

Note: ${ }^{*} \mathrm{p}<0,0.05$; compared with the control group, ${ }^{\#} \mathrm{p}<0.05$

TABLE 4: COMPARISON OF THE TWO GROUPS OF $\beta$-EP AND 5-HT ( $\overline{\mathrm{x}} \pm \mathbf{s}, \mathrm{ng} / \mathrm{l})$

\begin{tabular}{lcccc}
\hline Group & Number of cases & Time & B-EP & 5-HT \\
\hline \multirow{2}{*}{ Control group } & \multirow{2}{*}{50} & Before treatment & $95.36 \pm 18.02$ & $0.96 \pm 0.32$ \\
& & After treatment & $137.22 \pm 26.35^{*}$ & $0.67 \pm 0.27^{*}$ \\
Observation group & \multirow{2}{*}{50} & Before treatment & $94.05 \pm 19.63$ & $0.93 \pm 0.36$ \\
& & After treatment & $162.85 \pm 29.41^{* \#}$ & $0.56 \pm 0.24^{* \#}$ \\
\hline
\end{tabular}

Note: ${ }^{*} \mathrm{p}<0,0.05$; compared with the control group, ${ }^{*} \mathrm{p}<0.05$ 


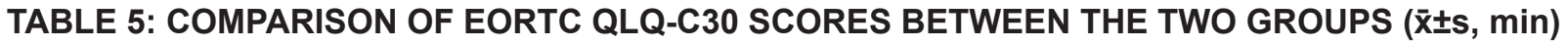

\begin{tabular}{|c|c|c|c|c|c|}
\hline \multirow{2}{*}{ Score } & & \multicolumn{2}{|c|}{ Control group $(n=50)$} & \multicolumn{2}{|c|}{ Observation group $(n=50)$} \\
\hline & & Before treatment & After treatment & Before treatment & After treatment \\
\hline $\begin{array}{l}\text { General health } \\
\text { field }\end{array}$ & & $35.52 \pm 5.69$ & $41.69 \pm 5.33^{*}$ & $34.97 \pm 5.82$ & $43.96 \pm 4.71^{* \#}$ \\
\hline \multirow[t]{4}{*}{ Functional area } & Physical function & $42.36 \pm 5.02$ & $46.66 \pm 4.78^{*}$ & $42.16 \pm 5.81$ & $50.33 \pm 4.69^{* \#}$ \\
\hline & Role function & $51.44 \pm 5.23$ & $56.02 \pm 3.89^{*}$ & $50.89 \pm 5.83$ & $59.91 \pm 4.17^{* \#}$ \\
\hline & Emotional function & $46.36 \pm 6.05$ & $52.69 \pm 4.72^{*}$ & $45.98 \pm 5.74$ & $59.36 \pm 5.57^{* \#}$ \\
\hline & Cognitive function & $58.63 \pm 5.63$ & $65.32 \pm 4.52^{*}$ & $58.96 \pm 5.99$ & $70.14 \pm 4.63^{* \#}$ \\
\hline \multirow{7}{*}{ Functional area } & Social function & $52.36 \pm 4.69$ & $58.96 \pm 4.45^{*}$ & $52.17 \pm 5.06$ & $63.74 \pm 3.99^{* \#}$ \\
\hline & Tired & $31.52 \pm 3.63$ & $30.58 \pm 3.85$ & $30.98 \pm 4.05$ & $29.92 \pm 3.69$ \\
\hline & Pain & $28.65 \pm 3.14$ & $25.45 \pm 3.01^{*}$ & $28.55 \pm 3.36$ & $22.96 \pm 2.57^{* \#}$ \\
\hline & Feel sick and vomit & $28.25 \pm 4.02$ & $30.69 \pm 3.85^{*}$ & $28.17 \pm 3.96$ & $33.15 \pm 3.16^{* \#}$ \\
\hline & Shortness of breath & $24.69 \pm 3.36$ & $24.45 \pm 4.32$ & $24.71 \pm 4.05$ & $25.01 \pm 4.17$ \\
\hline & Insomnia & $33.25 \pm 4.15$ & $27.25 \pm 3.21^{*}$ & $33.34 \pm 4.74$ & $24.66 \pm 2.73^{* \#}$ \\
\hline & Loss of appetite & $41.22 \pm 4.03$ & $35.23 \pm 3.66^{*}$ & $41.09 \pm 4.28$ & $32.88 \pm 3.79^{* \#}$ \\
\hline \multirow{3}{*}{ Symptomatic areas } & Constipation & $28.52 \pm 3.96$ & $27.96 \pm 4.12$ & $28.46 \pm 3.96$ & $28.02 \pm 3.24$ \\
\hline & Diarrhea & $27.96 \pm 3.54$ & $27.89 \pm 3.58$ & $28.02 \pm 3.88$ & $28.23 \pm 3.24$ \\
\hline & $\begin{array}{l}\text { Economic } \\
\text { difficulties }\end{array}$ & $36.63 \pm 5.88$ & $36.99 \pm 5.41$ & $36.89 \pm 4.58$ & $26.56 \pm 5.27$ \\
\hline
\end{tabular}

Note: ${ }^{*} p<0,0.05$; compared with the control group, ${ }^{*} p<0.05$

Safety analysis was also done. The observation group had nausea and vomiting in 6 cases, dizziness in 3 cases, drowsiness in 1 case, a total of 10 cases, the incidence was $20.00 \%$. The control group had nausea and vomiting in 3 cases, dizziness in 1 case, 1 case of drowsiness, a total of 5 cases, and the incidence of $10.00 \%$. The adverse reactions of the two groups were similar $(\mathrm{p}>0.05)$.

The mechanism of cancer pain is complicated, which is related to the pain caused by tumor itself and the pain caused by tumor treatment. Patients with bone metastasis in lung cancer need to suffer from severe cancer pain. Pain can cause negative emotions, sleep disorders and decreased treatment compliance. The above results can increase the sensitivity of the body to pain, further aggravate cancer pain, so as to form a vicious circle, which not only affects the compliance of anti-cancer treatment of patients with bone metastasis of lung cancer, but also leads to a decline in quality of life ${ }^{[8]}$. Therefore, the correct assessment and control of cancer pain is of great significance for patients with bone metastasis of lung cancer. Dezocine is a commonly used drug in the clinical treatment of cancer pain. It can completely excite the $\kappa$ receptor, weaken the influence on the $\mu$ receptor and do not lead to addiction. It has the advantages of rapid onset and less adverse reactions. Intramuscular injection of dezocine can avoid stimulating the gastrointestinal tract, especially for patients who cannot eat ${ }^{[9]}$.
Oxycodone hydrochloride is an opioid receptor agonist directly acting on the central nervous system. Its analgesic effect is stronger than that of morphine and the analgesic effect has no dose-capping effect. Oxycodone hydrochloride has the advantages of strong analgesic effect, rapid onset and lasting effect, but it has many adverse reactions and withdrawal syndrome can occur when sudden interruption or reduction occurs ${ }^{[10]}$. Cui Jian et al. applied oxycodone hydrochloride sustainedrelease tablets in outpatient treatment of moderate and severe adult cancer pain and found that it had better relief effect on visceral pain and good safety ${ }^{[11]}$. In this study, dezocine combined with oxycodone was applied to the treatment of severe cancer pain in bone metastasis of advanced lung cancer. The NRS score was used to evaluate the degree of pain. It was found that dezocine combined with oxycodone had better short-term analgesic effect in the treatment of severe cancer pain in bone metastasis of advanced lung cancer, which could better reduce the number of pain outbreaks in $24 \mathrm{~h}$, shorten the first stable time of pain control and prolong the time of sleep increase in $24 \mathrm{~h}$. This is because oxycodone hydrochloride sustained-release tablets have two release modes: immediate release and controlled release. Among them, $38 \%$ of the components rapidly produce analgesic effect after oral administration for 1 $\mathrm{h}$ and $62 \%$ of the components slowly release within 12 $\mathrm{h}$, playing a continuous analgesic effect, so that patients cancer pain can be better controlled and help patients sleep. After better rest, the patients emotional state improved and his tolerance to pain increased ${ }^{[12]}$. 
NRS score is a subjective pain assessment tool and its final results are affected by many factors such as patient tolerance and understanding ${ }^{[13]}$. The laboratory pain related factors have certain objectivity in indicating the degree of pain. $\beta$-EP is a kind of morphine hormone secreted by the pituitary gland, which can produce pleasure and analgesic effect $^{[14]}$. 5-HT can cause blood vessels, smooth muscle contraction, involved in pain formation and transmission process $^{[15]}$. In this study, the levels of $\beta$-EP and 5-HT in the two groups before and after treatment were detected. It was found that dezocine combined with oxycodone in the treatment of severe cancer pain in advanced lung cancer with bone metastasis can increase the level of endogenous analgesic factor $\beta$-EP, reduce the level of pain causing factor $\beta$-EP and regulate the expression of pain factors.

In this study, EORTC QLQ-C30 was used to evaluate the quality of life of patients in the two groups. It was found that dezocine combined with oxycodone in the treatment of severe cancer pain caused by bone metastasis of advanced lung cancer could better improve the quality of life of patients, which was related to the better relief of pain, insomnia and loss of appetite in patients receiving this treatment. The study also found that the incidence of nausea and vomiting, dizziness, drowsiness and other adverse reactions in patients treated with dezocine combined with oxycodone was higher than that in patients treated with dezocine alone. However, the statistical analysis results of the two groups showed similar adverse reactions. This may be related to the bias caused by the small sample size of this study. In the future, large sample studies should be accumulated to explore whether dezocine combined with oxycodone regimen will increase the risk of adverse reactions.

In summary, dezocine combined with oxycodone in the treatment of severe cancer pain caused by bone metastasis of advanced lung cancer can effectively relieve pain, regulate the expression of pain factors and improve the quality of life but the adverse reactions should be observed. In clinical application, attention should be paid in balancing the advantages and disadvantages between analgesia and adverse reactions and oxycodone should be used in appropriate way.

\section{Conflicts of interest:}

The authors declared no conflict of interest.

\section{REFERENCES}

1. Washington KT, Oliver DP, Smith JB, Kruse RL, Meghani SH, Demiris G. A Comparison of rural and urban hospice family care givers cancer pain knowledge and experience. J Pain Symptom Manage 2019;58(4):685-9.
2. Loeffen EA, Kremer LC, van de Wetering MD, Mulder RL, Font-Gonzalez A, Dupuis LL, et al. Reducing pain in children with cancer: Methodology for the development of a clinical practice guideline. Pediatr Blood Cancer 2019;66(6):e27698.

3. Brant JM. Assessment and management of cancer pain in older adults: strategies for success. Asia Pac J Oncol Nurs 2018;5(3):248-53.

4. Ngamkham S, Holden JE, Smith EL. A systematic review: mindfulness intervention for cancer-related pain. Asia Pac J Oncol Nurs 2019;6(2):161-9.

5. Csco CS. Chinese Society of Clinical Oncology (CSCO) diagnosis and treatment guidelines for colorectal cancer 2018 (English version). Chin J Cancer Res 2019;31(1):117-34.

6. Zhou X, Qiu GQ, Bao WA, Zhang DH. The prognostic role of nutrition risk score (NRS) in patients with metastatic or recurrent esophageal squamous cell carcinoma (ESCC). Oncotarget 2017;8(44):77465-73.

7. National Health Commission of the People's Republic of China. Chinese guidelines for diagnosis and treatment of prostate cancer 2018 (English version). Chin J Cancer Res 2019;31(1):67-83.

8. Kim KS, Loring S, Kwekkeboom K. Use of art-making intervention for pain and quality of life among cancer patients: a systematic review. J Holist Nurs 2018;36(4):341-53.

9. Jenkins BN, Roemer RJ, Martinez A, Torres TK, Fortier MA. Cold pressor pain response in children with cancer. J Pediatr Hematol Oncol 2018;40(5):368-73.

10. Sipila R, Hintsa T, Lipsanen J, Tasmuth T, Estlander AM, Kalso E. The relationship between anger regulation, mood, pain, and pain-related disability in women treated for breast cancer. Psychooncology 2019;28(10):2002-8.

11. Ishida K, Ohnuki K, Usami S, Komatsu H, Sasaki A. A survey on the current status of clinical resources for diagnosis and treatment of breast cancer in rural hospitals of the Tohoku region in Japan. Breast Cancer 2021;28(1):161-7.

12. Ulas S, Eyigor S, Caramat I. Quality of life and neuropathic pain in hospitalized cancer patients: a comparative analysis of patients in palliative care wards versus those in general wards. Indian J Palliat Care 2018;24(3):325-33.

13. Nawi RI, Chui PL, Ishak WZ, Chan CM. Oral Cryotherapy: Prevention of oral mucositis and pain among patients with colorectal cancer undergoing chemotherapy. Clin J Oncol Nurs 2018;22(5):555-60.

14. Jin PC, Gou B, Qian W. Urinary markers in treatment monitoring of lung cancer patients with bone metastasis. Int J Biol Markers 2019;34(3):243-50.

15. Soran A, Dogan L, Isik A, Ozbas S, Trabulus DC, Demirci U, et al. The effect of primary surgery in patients with De Novo stage IV breast cancer with bone metastasis only (Protocol BOMET MF 14-01): A multi-center, prospective registry study. Ann Surg Oncol 2021:1-10.

This is an open access article distributed under the terms of the Creative Commons Attribution-NonCommercial-ShareAlike 3.0 License, which allows others to remix, tweak, and build upon the work non-commercially, as long as the author is credited and the new creations are licensed under the identical terms

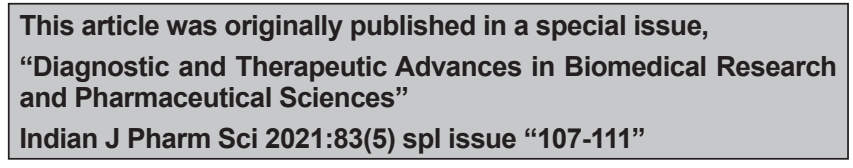

